\title{
ADAM9: A novel player in vestibular schwannoma pathogenesis
}

\author{
MARIA BREUN ${ }^{1}$, ALEXANDRA SCHWERDTFEGER ${ }^{1}$, DONATO DANIEL MARTELLOTTA ${ }^{1}$, \\ ALMUTH F. KESSLER ${ }^{1}$, CAMELIA M. MONORANU ${ }^{2}$, CORDULA MATTHIES $^{1}$, \\ MARIO LÖHR ${ }^{1 *}$ and CARSTEN HAGEMANN ${ }^{1 *}$ \\ ${ }^{1}$ Department of Neurosurgery, University Hospital Würzburg; ${ }^{2}$ Department of Neuropathology, \\ Institute of Pathology, University of Würzburg, D-97080 Würzburg, Germany
}

Received April 27, 2019; Accepted October 2, 2019

DOI: $10.3892 / 01.2020 .11299$

\begin{abstract}
A disintegrin and metalloproteinase 9 (ADAM9) is a member of the transmembrane ADAM family. It is expressed in different types of solid cancer and promotes tumor invasiveness. To the best of our knowledge, the present study was the first to examine ADAM9 expression in vestibular schwannomas (VS) from patients with and without neurofibromatosis type 2 (NF2) and to associate the data with clinical parameters of the patients. The aim of the present study was to evaluate if ADAM9 could be used as prognostic marker or therapeutic target. ADAM9 mRNA and protein levels were measured in
\end{abstract}

Correspondence to: Dr Maria Breun, Department of Neurosurgery, University Hospital Würzburg, Josef-Schneider-Straße 11, D-97080 Würzburg, Germany

E-mail: breun_m@ukw.de

Abbreviations: ADAM, a disintegrin and metalloproteinase; Akt, protein kinase $\mathrm{B}$; bp, base pair; $\mathrm{CCC}$, comprehensive cancer center; CD44, cell adhesion molecule 44; CD68, cell adhesion molecule 68; cDNA, complementary desoxyribonucleic acid; $\mathrm{Cy} 2 / 3$, cyanine $2 / 3$; $\mathrm{dB}$, decibel; ECL, enhanced chemiluminescence; EGF, epidermal growth factor; Erb/Her4, erythroblastic oncogene; FAM, fluorescein amidite; FERM, 4.1 ezrin/radixin/moesin protein; FGF-R, fibroblast growth factor receptor; Grb, growth factor receptor-bound protein; HRP, horseradish peroxidase; $\mathrm{Hx}$, hearing class according to the Hannover Classification; IgG, immunoglobulin G; IHC, immunohistochemistry; IP3, inositol trisphosphate; MAP/MEK, mitogen-activated ERK kinase; MMP, matrix metalloproteinase; NF2, neurofibromatosis; PI3, phosphoinositide 3-kinase; proHB-EGF, pro-heparine binding epidermal growth factor; $\mathrm{PKC}$, protein kinase $\mathrm{C}$; qPCR, quantitative polymerase chain reaction; r.p., rapidly progressive;S100, Schwann cell marker protein 100; SH3, src homology 3; Src, sarcoma; s.p., slowly progressive; TIMP, tissue inhibitor of matrix metalloproteinase; TNF $\alpha$, tissue necrosis factor $\alpha$; Tx, tumor extension according to the Hannover Classification; VEGF, vascular endothelial growth factor; VIC, 2'-chloro-7'phenyl-1,4-dichloro-6-carboxy-fluorescein; VS, Vestibular schwannoma; WB, western blotting

*Contributed equally

Key words: vestibular schwannoma, a disintegrin and metalloproteinase 9, pathogenesis, molecular marker
VS samples $(n=60)$. A total of 30 of them were from patients with neurofibromatosis. Healthy peripheral nerves from autopsies $(n=10)$ served as controls. ADAM9 mRNA levels were measured by PCR, and protein levels were determined by immunohistochemistry (IHC) and western blotting (WB). The Hannover Classification was used to categorize tumor extension and hearing loss. ADAM9 mRNA levels were 8.8 -fold higher in VS compared with in controls. The levels were 5.6-fold higher in patients with NF2 and 12-fold higher in patients with sporadic VS. WB revealed two mature isoforms of the protein, and according to IHC ADAM9 was mainly expressed by S100-positive Schwann cells. There was a strong correlation between ADAM9 mRNA expression and the level of functional impairment $(r \sim 1, p=0.01)$. Particularly, the secreted isoform of ADAM9 was expressed in patients with higher hearing impairment. ADAM9 mRNA was overexpressed in the tumor samples relative to healthy vestibular nerves, and there was an association between higher ADAM9 expression levels and greater hearing impairment. Therefore, ADAM9 may be a prognostic marker for VS, and ADAM9 inhibition might have the potential as a systemic approach for the treatment of VS.

\section{Introduction}

Vestibular schwannomas (VS) are benign nerve sheath tumors of the vestibular nerve that arise from neoplastic Schwann cells $(1,2)$. These tumors usually appear sporadically, but in rare cases $(1: 33,000)$ they are part of a genetic disorder, called neurofibromatosis type 2 . NF2 is associated with the loss of the NF2 gene on chromosome 22, which encodes for merlin, a tumor suppressor protein $(3,4)$. NF2 patients develop a multitude of tumors like meningeomas, ependymomas and as the hallmark tumors bilateral VS. These tumors usually have a higher recurrence rate, grow faster, and are much more adherent to the surrounding structures compared to their sporadic counterparts (5). Therefore, they are often associated with persistent cranial nerve deficits and sole surgery is not a long-lasting solution in these cases-in contrast to sporadic VS. Thus, an efficacious systemic therapy is urgently needed.

The main known pathomechanism for vestibular schwannoma is the loss of function by Merlin. Merlin is a 4.1 protein/ezrin/radixin/moesin protein (FERM) that connects the cytoskeleton with the cell membrane. It is activated by the cells' attachment to the extracellular matrix and by 
intercellular adhesion (6). Merlin's loss of function is the main known mechanism for the development of VS and results in the activation of two signaling pathways. These are the Ras/Raf/MEK pathway and the PI3K/Akt/mTOR pathway, which inhibit apoptosis and result in higher cell survival or proliferation rates $(3,7,8)$. Furthermore, the Hippo pathway and the VEGF-mediated signaling pathway, are also activated by Merlin's loss of function $(3,6)$. Indeed, the VEGF-inhibitor Bevacizumab has been shown to effectively target VEGF overexpression in individual cases of NF2 associated VS (3), but only for a short period in the majority of patients. To date, there is no effective systemic treatment option available for VS in terms of maintaining a stable disease state or even inducing tumor shrinkage. Therefore, there is a huge necessity to identify useful molecular therapeutic targets, especially for patients with NF2 $(3,9)$.

Members of the A-Disintegrin and Metalloproteinase protein (ADAM) family are therapeutic targets for many tumor entities. The ADAM protein family consists of 21 functional transmembrane proteins with 8 transmembrane domains. These include a signal peptide, a propeptide, metalloproteinase activity, a disintegrin sequence, a cysteine-rich region, an EGF-like domain, a transmembrane part and a cytoplasmic tail $(10,11)$. One member of this protein family is ADAM9, which is encoded on chromosome 8 and was first described in $1996(12,13)$. It plays a role in cell-adhesion and cell-signaling and is overexpressed in several cancers like breast-, non-small cell lung-, pancreas-, stomach-, hepatocellular-cancer, melanoma and glioblastoma (10,14-17).

Two mature isoforms are produced by alternative mRNA splicing, a shorter, secreted and a larger, transmembrane version. The first one derives from a $68 \mathrm{kDa}$ precursor and has a mature size of $47 \mathrm{kDa}$ while the latter is represented by a $114 \mathrm{kDa}$ precursor and matures as $84 \mathrm{kDa}(11,18,19)$. Functional differences of these isoforms are subject of recent research. Especially the secreted isoform promotes cell migration and is correlated with a higher tumor invasiveness by modulating the interaction with integrins $(10,11,14,18)$. One further function of ADAM9 is protein-shedding of proHB-EGF by its proteinase activity, which is induced by PKC. Furthermore, ADAM9 is a ligand for integrins $\alpha 2 \beta 1, \alpha 6 \beta 4$ and others $(10,14)$. ADAM proteins contain a SH3-binding site, which can activate SH3-domain-containing-signalling molecules like Src and Grb. In contrast to other ADAM family members, ADAM9 cannot be specifically inhibited $(10,11,14,20)$. Importantly, ADAM9 overexpression is observed under oxidative stress with increased levels of reactive oxygen species, which are regularly generated by cancer cells $(21,22)$.

Recently, we demonstrated that CXCR4 is of relevance for VS pathogenesis (23). Therefore, we assumed that other proteins beside Merlin and CXCR4 also might be involved. To the best of our knowledge, no data on ADAM9 expression in VS have been published so far. We hypothesized that ADAM9 could be overexpressed in VS because of its role in other solid cancers and its similarity to the function of Merlin. Furthermore, ADAM9 could be a suitable therapeutic target for the treatment of VS, as it is already therapeutically targeted in other cancer types and in Alzheimer's disease (24). Therefore, we evaluated its potential role as a prognostic marker for VS by examining its expression and distribution in sporadic and NF2- associated VS tissue and its correlation to clinical parameters of the patients, especially their hearing-loss.

\section{Materials and methods}

Tissue samples. The study was approved by the local ethics committee of the University Hospital Würzburg with the statement number 145/16 and written informed consent was obtained from the patients for the use of their tissue. Tissue collection was from 2010-2015. Directly after surgical excision, the tissue was divided in half: one half was cryopreserved, and the other half was fixed in formalin. All tumor samples were neuropathologically assessed according to WHO-criteria (2). Typical for Antoni type A tissue is the high cell density in a spindle-shaped arrangement, and for Antoni type $\mathrm{B}$ tissue a loose meshwork of gelatinous and microcystic tissue is specific (25). Mixed types, called Antoni A/B, are also common. As controls served 6 normal vestibular nerves that were obtained from autopsies within the first $24 \mathrm{~h}$ after death and 4 sural nerves from biopsies.

Clinical parameters. Hearing function and tumor extension were categorized using the Hannover Classification $(26,27)$. Tone and speech audiometry were measured several days before surgery to estimate hearing function. Hearing deterioration was categorized into 6 classes in $20 \mathrm{~dB}$ steps: $\mathrm{H} 1$ has a maximum of $20 \mathrm{~dB}$ hearing loss in tone audiometry and a similar good result in speech audiometry, which is a nearly normal hearing function. H6 means deafness, with at least $100 \mathrm{~dB}$ hearing loss and no speech discrimination (Table I). The tumor extension into the cerebellopontine angle was estimated as follows: $\mathrm{T} 1$ is an intrameatal tumor, $\mathrm{T} 2$ is an intra- and extrameatal tumor, a T3A tumor fills the cerebellopontine cistern, a T3B tumor has contact to the brainstem, a T4A tumor compresses the brainstem, and a T4B tumor already dislocates the brain stem (Table II).

Tumor growth dynamics were categorized by magnetic resonance imaging (MRI) performed during a 'watch and wait' period before surgery. The histological proliferation rate was determined according to Ki67 staining. Both groups comprised 15 slowly growing VS (growth rate of less than $2 \mathrm{~mm}$ per year or $<2 \% \mathrm{Ki} 67$-positive cells) and 15 tumors, which were faster growing (growth rate over $2 \mathrm{~mm}$ per year or $>2 \%$ Ki67-positive cells) $(28,29)$.

$m R N A$ and protein extraction. NucleoSpin RNA/Protein kit (Macherey-Nagel) was used to purify protein and mRNA from $30 \mathrm{mg}$ tissue samples according to the manufacturer's instructions. Utilizing the Qubit 2.0 Fluorometer (Thermo Fisher Scientific, Inc.) the concentrations of the extracted mRNA and of the isolated protein were measured. Reverse transcription of the mRNA to cDNA was performed by the High-capacity RNA-to-cDNA kit (Applied Biosystems) and the T3000 Thermocycler (Biometra). The isoloated cDNA samples were stored at $-80^{\circ} \mathrm{C}$.

Reverse transcription-quantitative PCR (RT-qPCR). The StepOnePlus Real-Time PCR System (Applied Biosystems) was used for analysis of the ADAM9 mRNA expression in VS and control nerve samples. The cDNA concentration 
Table I. Hannover Classification of audiometry results.

\begin{tabular}{lcc}
\hline Class & PTA result $(\mathrm{dB})$ & Speech discrimination score $(\%)$ \\
\hline $\mathrm{H} 1$ & $0-20 \mathrm{~dB}$ & $100-95$ \\
$\mathrm{H} 2$ & $21-40 \mathrm{~dB}$ & $95-70$ \\
$\mathrm{H} 3$ & $41-60 \mathrm{~dB}$ & $65-40$ \\
$\mathrm{H} 4$ & $61-80 \mathrm{~dB}$ & $35-10$ \\
$\mathrm{H} 5$ & $80-100 \mathrm{~dB}$ & $5-0$ \\
H6 & $>100 \mathrm{~dB}$ & 0 \\
\hline
\end{tabular}

PTA, pure tone audiometry; $\mathrm{dB}$, decibel.

Table II. Hannover Classification of the tumor extension.

\begin{tabular}{cl}
\hline Class & \multicolumn{1}{c}{ Tumor extension } \\
\hline T1 & Purely intrameatal \\
T2 & Intra- and extrameatal \\
T3 & Filling the cerebellopontine cistern \\
T3A & Without brainstem contact \\
T3B & Reaching the brainstem \\
T4 & Brainstem compression \\
T4A & Compressing the brainstem \\
T4B & Dislocating the brainstem and compressing the \\
& fourth ventricle \\
\hline
\end{tabular}

was mixed with TaqMan Universal Master Mix (Applied Biosystems) after adjustment to the amount of sample with the lowest concentration. GAPDH-VIC PL (HS99999905_m1; 5'3' sequence: gggcgctggtcaccagggctgctt) was used as an internal control, and ADAM9_FAM (HS00177638_m1; 5'3' sequence: gtgccactgggaatgctttgtgtgg) assays (Applied Biosystems) were used to evaluate the relative ADAM9 expression in a duplex setting. PCR was running for $10 \mathrm{~min}$ at $95^{\circ} \mathrm{C}$ followed by 50 cycles of $15 \mathrm{sec}$ at $95^{\circ} \mathrm{C}$ and $60 \mathrm{sec}$ at $60^{\circ} \mathrm{C}$. All samples were done in triplicate. The data were analyzed utilizing the $2^{-\Delta \Delta \mathrm{Cq}}$ method and GAPDH was used as loading control for normalization (30).

Western blotting analysis. A total of $16 \mu 1$ pure protein lysate of the sample with the lowest concentration was used. A comparative amount of protein lysate from the other samples was diluted in water (in all $16 \mu \mathrm{l}$ volume) and used. These samples were mixed with sample buffer $(6.25 \mu \mathrm{l})$ and reducing agent $(2.5 \mu \mathrm{l})$, incubated at $70^{\circ} \mathrm{C}$ for $10 \mathrm{~min}$, and centrifuged for $1 \mathrm{~min}$ at $11,000 \mathrm{x} \mathrm{g.} 20 \mu \mathrm{l}$ of each sample were loaded onto a $4-12 \%$ polyacrylamide NuPage Bis-Tris gel (Invitrogen). Electrophoresis was running for $1 \mathrm{~h}$ at $200 \mathrm{~V}$ and $120 \mathrm{~mA}$ using the XCell SureLock system (Invitrogen). The transferring of the separated proteins to nitrocellulose membranes (Invitrogen) was done with the iBlot kit and system (Invitrogen) following the manufacturer's instructions. Blocking of the membrane was carried out with $5 \%$ nonfat milk powder (Roth) in TBST $(0.1 \%$ Tween 20$)$ at room temperature for $1 \mathrm{~h}$
Table III. Differences in $2^{-\Delta \Delta C q}$ values for NF or sporadic growth and their different dynamics.

\begin{tabular}{lcr}
\hline Tissue & Tumor growth dynamic & $2^{-\Delta \Delta C q}$ value \\
\hline NF VS & s.p. & 3.28 \\
\multirow{2}{*}{ Sporadic VS } & r.p. & 7.86 \\
& s.p. & 15.45 \\
& r.p. & 8.66 \\
\hline
\end{tabular}

The differences in the $2^{-\Delta \Delta C \mathrm{q}}$ values for the different growth dynamics were not significant $(\mathrm{P}>0.05)$. s.p., slow progression; r.p., rapid progression; NF, neurofibromatosis type 2; NF VS, NF-associated vestibular schwannoma; sporadic VS, sporadic vestibular schwannoma not associated with NF.

and probed with mouse polyclonal antibody 57934 against human ADAM9 (Abcam) at a dilution of 1:500 in TBST (GE Healthcare UK Limited) and with $\gamma$-tubulin antibody T6557 in a dilution of 1:5,000 in TBST (Sigma). The secondary antibody, sheep anti-mouse IgG-HRP NA931V (Amersham), was diluted in TBST $(1: 1,000)$. For protein detection the ECL Western Blotting Analysis System (Amersham) was used. Visual analysis was performed by two independent investigators, evaluating the presence or absence of specific protein bands.

Immunohistochemistry. $3 \mu \mathrm{m}$ sections were cut from formalin-fixed paraffin-embedded blocks of the VS tissue and stained with anti-ADAM9 antibody (Abcam) using a 1:800 dilution in dilution buffer (DCS). ADAM9 protein expression was visualized using a poly-link secondary antibody and a peroxidase kit (Dako; DCS Innovative Diagnostic Systems). Brown staining showed positive signals, and for counterstaining hematoxylin was used.

Immunofluorescence analysis was also performed on $3 \mu \mathrm{m}$ formalin-fixed paraffin sections. The slices were washed $2 \mathrm{x}$ $10 \mathrm{~min}$ in xylol followed by an ethanol series with decreasing concentrations for 5 min each in 100, 96, 70, and 50\% ethanol. Sections were blocked at a 1:2 dilution for $20 \mathrm{~min}$ with $10 \%$ goat serum (Life Technologies) in antibody dilution buffer (DCS). Double-staining was performed with anti-ADAM9 antibody (Abcam) at a 1:100 dilution in dilution buffer (DCS) and anti-S100 antibody (Abcam) at a 1:100 dilution or anti-CD68 (Dianova) at $1: 200$ at $4^{\circ} \mathrm{C}$ overnight. Protein expression was visualized with $\mathrm{Cy} 3$-anti-mouse (red, Dianova) at a dilution of 1:100 and Cy2-anti-rabbit (green, Dianova) at a dilution of 1:50 for $1 \mathrm{~h}$ at room temperature, both used as secondary antibodies. Fluoroshield mounting medium (Abcam) was used for the slides.

All immunohistochemically stained slides were analyzed using a light microscope (Leica). Liver tissue served as negative control and glioblastoma tissue as positive control for the staining experiments.

Statistical analysis. StepOne software v2.3 and ExpressionSuite Software v1.04 (Thermo Fisher Scientific, Inc.) was used for mRNA expression analysis. GAPDH mRNA expression 
Table IV. Numbers of patients with NF VS and sporadic VS according to their clinical features.

\begin{tabular}{|c|c|c|c|c|c|c|c|c|c|}
\hline \multirow[b]{2}{*}{ Tissue } & \multirow[b]{2}{*}{ Tumor growth dynamic } & \multirow{2}{*}{$\begin{array}{c}\text { Tumor extension } \\
\leq \mathrm{T} 3 \mathrm{~A}\end{array}$} & \multicolumn{4}{|c|}{ Hearing function } & \multicolumn{3}{|c|}{$\begin{array}{l}\text { Histological/ } \\
\text { Antoni type }^{\mathrm{a}}\end{array}$} \\
\hline & & & $\geq \mathrm{T} 3 \mathrm{~B}$ & $\mathrm{H} 1 / 2$ & $\mathrm{H} 3 / 4$ & $\mathrm{H} 5 / 6$ & A & B & $\mathrm{A} / \mathrm{B}$ \\
\hline \multirow[t]{2}{*}{ NF VS } & s.p. & 4 & 11 & 4 & 7 & 4 & 5 & 0 & 9 \\
\hline & r.p. & 1 & 14 & 6 & 1 & 8 & 4 & 0 & 11 \\
\hline \multirow[t]{2}{*}{ Sporadic VS } & s.p. & 4 & 12 & 3 & 8 & 4 & 7 & 0 & 8 \\
\hline & r.p. & 6 & 8 & 5 & 6 & 4 & 6 & 2 & 7 \\
\hline
\end{tabular}

${ }^{a}$ The histological Antoni type was not known in all cases. Tumor extension and hearing function according to the Hannover classification are shown along with the respective histological subtype. s.p., slow progression; r.p., rapid progression; NF, neurofibromatosis type 2; NF VS, NF-associated vestibular schwannoma; sporadic VS, sporadic vestibular schwannoma not associated with NF.

was analyzed to normalize the data. Statistical analysis was performed with Microsoft Exel 2010 with XLSTAT(Redmond). Normality was tested by Shapiro-Wilk test. Statistical significance was determined using Mann-Whitney-U-test and the Kruskal Wallis test. $\mathrm{P}<0.05$ was considered to indicate a statistically significant difference. Correlation was evaluated using the Pearson correlation coefficient.

\section{Results}

Patient cohort. $60 \mathrm{VS}$ tumor samples from 58 patients (32 women, 26 men; mean age, 42 years) were analyzed in this study. Of the 60 samples, half were obtained from patients with NF2 (16 women, 14 men) and the other half from patients with sporadic VS (18 women, 12 men). The mean age of the control group was 57 years, the mean age of the sporadic vestibular schwannoma group was 51 years. This is not a significant difference. However, patients with neurofibromatosis are much younger at tumor manifestation and time of surgery compared to patients with sporadic vestibular schwannoma.

mRNA expression of ADAM9 in VS. In comparison to the control group a mean 8.8 times overexpression of ADAM9 mRNA ( $\mathrm{SD}=13 ;$ median $=3.4 ; 95 \%$ confidence interval $(\mathrm{CI})=5.5-12.1)$ was detected in all VS combined. The subgroup of sporadic VS displayed a mean 12 times overexpression of ADAM9 (SD = 13; median $=5.2$; 95\% CI=7.4-16.7) (Fig. 1). VS with NF2 background had a mean 5.6 times overexpression of ADAM9 vs. the control group ( $\mathrm{SD}=12.5$; median=2.2; 95\% CI=1.1-10.0) (Fig. 1). The ADAM9 expression was significantly different between the two subgroups (Mann-Whitney U test, $\mathrm{P}<0.019$ ). The growth velocity showed no correlation with ADAM9 expression levels, but there was a trend $(\mathrm{P}=0.107)$ : Rapidly progressive tumors $(\mathrm{Ki} 67>2 \%)$ had higher ADAM9 expression in NF2 cases, while the opposite was observed in sporadic VS. In these cases, higher ADAM9 expression levels were found in the slowly progressive tumors (Table III). Tumor extension (Table IV) at surgery also did not significantly correlate with ADAM9 mRNA-expression levels $(\mathrm{P}=0.15)$.

Importantly, the impairment of the hearing function (18x H1/2, 22x H3/4 and 20x H5/6 according to Hannover Classification) correlated strongly with ADAM9 expression
Table V. Numbers of patients according to tumor growth dynamic and NF background as well as staining intensity of the different isoforms in western blotting.

\begin{tabular}{lccc}
\hline Tissue & $\begin{array}{c}\text { Tumor growth } \\
\text { dynamic }\end{array}$ & $\begin{array}{c}68 \mathrm{kDa}, \mathrm{n} \\
\text { (yes/no) }\end{array}$ & $\begin{array}{c}95 \mathrm{kDa}, \mathrm{n} \\
\text { (yes/no) }\end{array}$ \\
\hline NF VS & s.p. & $2 / 13$ & $10 / 5$ \\
Sporadic VS & r.p. & $6 / 9$ & $9 / 6$ \\
& s.p. & $5 / 10$ & $7 / 8$ \\
& r.p. & $3 / 12$ & $7 / 8$ \\
\hline
\end{tabular}

s.p., slow progression; r.p., rapid progression; NF, neurofibromatosis type 2; NF VS, NF-associated with vestibular schwannoma; sporadic VS, sporadic vestibular schwannoma not associated with NF.

(Pearson correlation coefficient $\mathrm{r} \sim 1$ ). Patients with normal or good hearing function had a low ADAM9 expression (mean 3.8 fold; $\mathrm{SD}=5.9$; median=2.1; 95\% $\mathrm{CI}=1.2-6.7$ ) versus patients with medium or high hearing impairment, who had a much higher ADAM9 expression (mean 11.3 fold; $\mathrm{SD}=14.7$; median=3.5; 95\% CI=6.5-15.3). Differences were statistically significant (Mann-Whitney U Test, p=0.027) (Fig. 2).

Protein expression of ADAM9 in VS. To confirm the ADAM9 overexpression in VS on protein level Western blots were performed (Fig. 3A). Three ADAM9 isoforms of approximately 95, 84 and $68 \mathrm{kDa}$ were detectable (Fig. 3A), corresponding to the two precursor proteins and the mature $84 \mathrm{kDa}$ transmembrane form. The $68 \mathrm{kDa}$ isoform was detectable in fewer cases than the $95 \mathrm{kDa}$ isoform, but there was no significant difference of distribution between cases with and without NF2 background (Table V). There was low ADAM9 expression detectable in $50 \%$ of the control group and $50 \%$ had no ADAM9 expression.

Importantly, there was a correlation of the secreted ADAM9 isoform ( $68 \mathrm{kDa}$ ) expression detected by Western blot (Fig. 3B) and the grade of hearing impairment. Patients with good hearing function showed in two of 18 cases an expression of the $68 \mathrm{kDa}$ isoform, patients with medium hearing impairment in 5 of 22 cases and deaf patients in 9 of 20 cases. 
Table VI. ADAM9 staining results. Number of patients with weak/no staining or strong staining for ADAM9 according to the histological type. Only a distribution in type A and B regions were considered for analysis. Not for all histological regions staining results were available.

NF VS, n

Sporadic VS, n

Histological type

Weak/no staining

Strong staining

Weak/no staining

Strong staining

Type A region 17

3 25 1

Type B region 3

11

6

11

ADAM9, a disintegrin and metalloproteinase 9; NF, neurofibromatosis type 2; NF VS, NF-associated with vestibular schwannoma; sporadic VS, sporadic vestibular schwannoma not associated with NF.

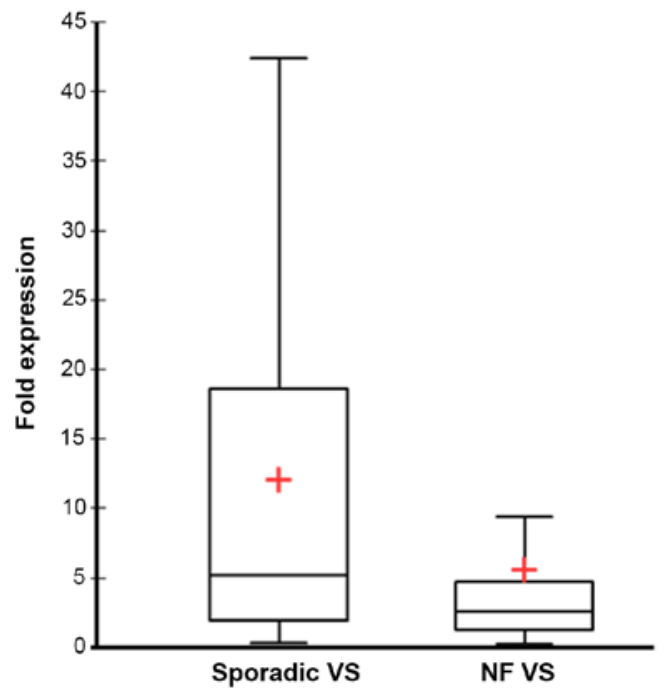

Figure 1. ADAM9 mRNA expression in the vestibular schwannoma subgroups. ADAM9 mRNA expression was analyzed by quantitative PCR using the $2^{-\Delta \Delta C q}$ method in the sporadic VS $(n=30)$, NF VS $(n=30)$ and in healthy sensory nerves $(n=10)$. Expression of the healthy nerves was set as 1. The red cross marks the mean, the bars show the 95\% CI. ADAM9, a disintegrin and metalloproteinase 9; NF VS, NF2-associated vestibular schwannoma; sporadic VS, sporadic vestibular schwannoma.

ADAM9 protein expression could be detected by immunohistochemistry of VS slices (Fig. 4). ADAM9 was localized predominantly membrane-bound and in the cytoplasm of Schwann cells.

Antoni type A regions, with a high cell density in a spindle shaped arrangement, showed weak or no ADAM9-staining in 42 of 46 cases. Antoni type B regions, which consist of a loose meshwork of gelatinous and microcystic tissue, were strongly immunopositive in 22 of 31 cases (Table VI). This strong relationship between ADAM9 expression and the histological architecture could be observed in sporadic as well as in NF2 associated VS.

Immunofluorescent double-staining revealed that ADAM9 was co-localized with the Schwann cell marker S100 in VS, but not in healthy nerves (Fig. 5). ADAM9 expression could only be detected within tumor cells while it did not co-localize with the macrophage marker CD68 (Fig. 5). Vestibular nerves

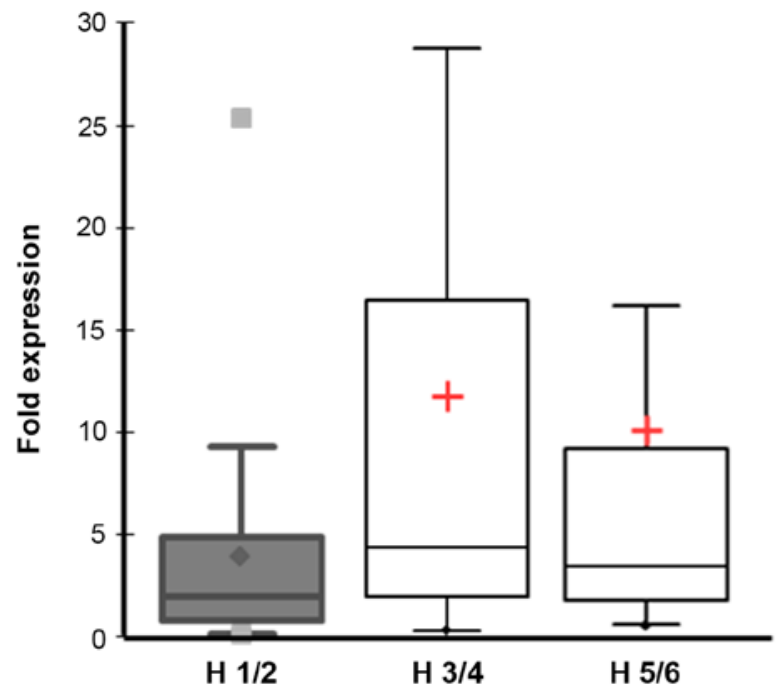

Figure 2. ADAM9 expression and hearing impairment. Hearing impairment caused by vestibular schwannoma was categorized according to the Hannover Classification. H1 indicates a maximum hearing loss of $20 \mathrm{~dB}$, $\mathrm{H} 2$ a loss of $40 \mathrm{~dB}, \mathrm{H} 3$ a loss of $60 \mathrm{~dB}, \mathrm{H} 4$ a loss of $80 \mathrm{~dB}, \mathrm{H} 5$ a loss of $100 \mathrm{~dB}$ and $\mathrm{H} 6$ a loss $>100 \mathrm{~dB}$. The difference in ADAM9 mRNA expression was statistically significant according to a Mann-Whitney $\mathrm{U}$ test $(\mathrm{P}=0.027)$. ADAM9, a disintegrin and metalloproteinase 9.

and sural nerves of the control group displayed a very low expression of ADAM9, and CD68 positive cells were only hardly found in these specimen.

\section{Discussion}

The present investigation demonstrates for the first time that ADAM9 is expressed in VS by neoplastic Schwann cells, and thus, could play a significant role in the pathogenesis of these tumors.

ADAM9 is involved in cell-cell and cell-matrix interaction by binding to several integrins and it promotes cell migration in tumor cells $(10,11)$. However, its specific function in tumor pathogenesis has not been elucidated at all so far $(10,14)$. For example, in breast cancer ADAM9 expression correlates with cancer progression (31), in prostate cancer it is a marker for relapse and in renal cancer it indicates a poor prognosis (11). In 

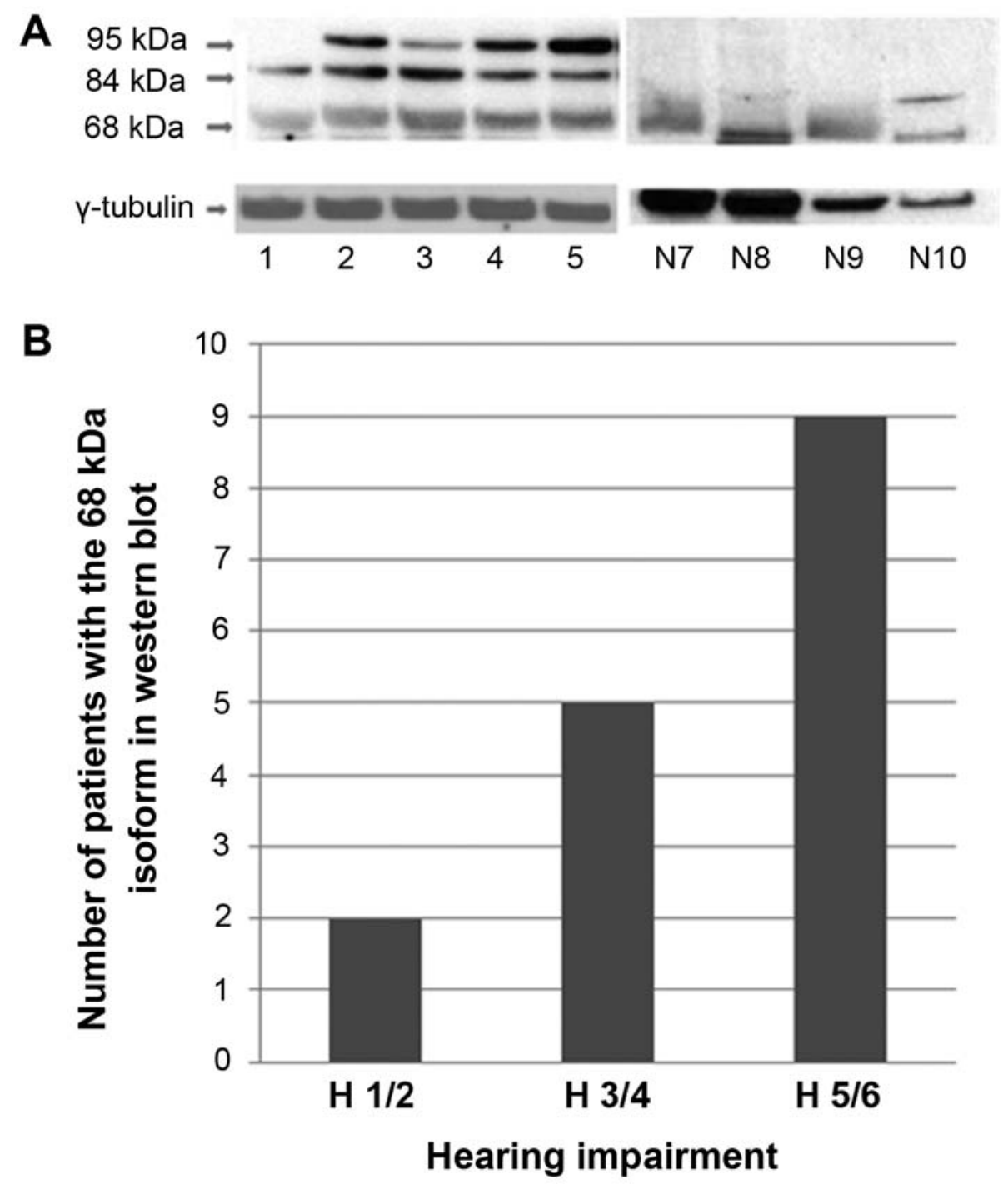

Figure 3. ADAM9 protein expression in VS. (A) Protein lysates were isolated from vestibular schwannoma samples and subjected to western blot analysis. ADAM9 isoforms of different sizes, as detected by a specific antibody, are indicated by arrows. $\gamma$-tubulin served as a loading control. Results are shown for 5 representative tumors (1-5) of the total cohort $(\mathrm{n}=60)$ and for 4 representative nerves (N7, 8, 9 and 10) for the control group ( $\mathrm{n}=10)$. (B) Expression of the $68 \mathrm{kDa}$ isoform of ADAM9 in WB was associated with the grade of hearing impairment ( $r>0.99)$ of the patients. ADAM9, a disintegrin and metalloproteinase 9; VS, vestibular schwannomas; $\mathrm{WB}$, western blotting.

prostate cancer blocking ADAM9 expression induced apoptotic cell death in vitro (11), and to treat pancreatic cancer, it is targeted by the matrix-metalloproteinase-inhibitor (CGS27023) (20).

ADAM protein family members are multifunctional proteins and process other transmembrane proteins like the TNF- $\alpha$-receptor, L-Selectine, CD44 and Erb4/Her4 by their proteolytic activity (11). This is called protein shedding. Other examples are the cleavage of EGF ligands and the FGF receptor, a key-mechanism during the genesis of prostate cancer (14). Protein shedding and cell migration enhance tumor invasion and are essential steps in tumor pathogenesis.

In the current study, ADAM9 overexpression in VS could be demonstrated by qPCR for the first time with a difference between NF2 and sporadic cases. The two subgroups were not completely comparable regarding the mean age. However, age as a confounding factor between sporadic and NF2 vestibular schwannoma, although possible, is unlikely in our opinion. ADAM9 showed its highest expression in slow growing sporadic VS, medium expression in rapidly growing tumors with and without NF2 and the lowest expression in slow growing VS with NF2 (Table I). ADAM9 overexpression did not correlate with the growth velocity or tumor extension, but with the functional impairment and therefore could be a marker for tumor invasiveness.

In daily clinical practice there is sometimes a mismatch between tumor extension and functional impairment in VS. Patients with a large tumor extension may have a good hearing function while those with small tumors may have a high hearing impairment (26). Although VS are histologically benign tumors, invasiveness is sometimes observed during surgery. Occasionally, the cranial nerves or even the brainstem appear to be infiltrated by the tumor. Therefore, invasive behavior could be an explanation for an over-proportional hearing loss in some cases with small tumors. Although invasive growth is more often seen in NF2-cases (5), growth velocity and tumor extension were not significantly different between the NF2and non-NF2-group analyzed here. Evaluating invasiveness was out of the scope of our study. Nevertheless, we reckon 


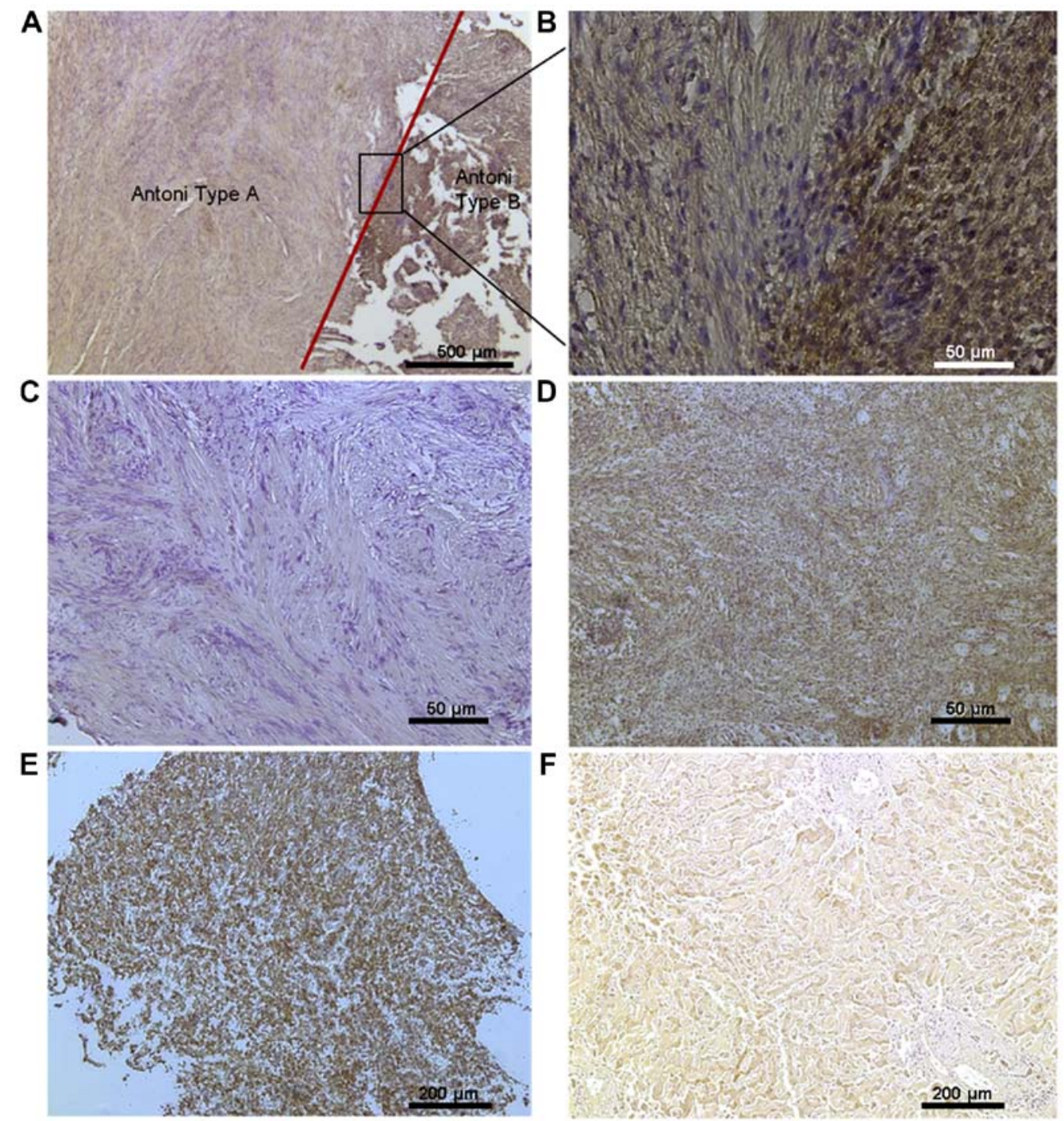

Figure 4. ADAM9 protein expression in VS. (A and B) Representative vestibular schwannoma with NF2 background. Antoni type A region with weak and Antoni type B region with strong ADAM9 expression, both are contained in the indicated region (B) of the indicated region in (A). Magnification, $\mathrm{x} 40$. (C) Representative sporadic vestibular schwannoma Antoni type A region with weak ADAM9 expression. (D) Antoni type B region with strong ADAM9 expression. (E) Glioblastoma tissue served as the positive control and (F) liver tissue as the negative control. ADAM9, a disintegrin and metalloproteinase 9; VS, vestibular schwannomas..

that the strong correlation between ADAM9 expression and functional impairment could be due to increased invasion of VS-cells in case of high ADAM9 expression.

This assumption is supported by the finding that Antoni type A schwannomas, which show a higher cell density and a more regular histological pattern, exhibited lower ADAM9 expression levels while Antoni type B tumors with a higher grade of degeneration (25) correlated with higher levels of ADAM9. Therefore, ADAM9 might be relevant for these histological differences and they are the correlate for tumor invasiveness. How ADAM9 could contribute to these histological changes is not known, perhaps its function as proteinase is important.

Different ADAM9-isoforms were detected by Western-blotting: The $95 \mathrm{kDa}$ band could represent the precursor-form of the mature $84 \mathrm{kDa}$ transmembrane isoform even though it was described with $114 \mathrm{kDa}$ in the literature $(11,18,19)$. The $68 \mathrm{kDa}$ band might be the precursor-form of the mature secreted protein. However, the latter was absent in our Western blot, perhaps because it is dissoluble. In breast cancer cells the larger 114/84 kDa isoform of ADAM9 was identified as suppressor of migration (19). On the other hand, the secreted $68 / 47 \mathrm{kDa}$ isoform, seems to be a promoter of migration in liver metastasis (18). In our cohort the larger $95 \mathrm{kDa}$ isoform was expressed in more cases than the secreted $68 \mathrm{kDa}$ isoform. Higher levels of the secreted ADAM9 isoform correlated strongly with the functional impairment of the patients. Thus, the secreted isoform, which was expressed in fewer cases, appeared to be an indicator for invasiveness in VS, as has been shown for migration in liver metastasis (18).

Further analysis of the isoform-expression depending on growth dynamics in NF2 and non NF2 as well as the comparison with the qPCR results were limited by the low number of only $n=15$ cases in each group. The growth velocities were not significantly different and ADAM9 therefore probably is not relevant for the different growth dynamics in cases with neurofibromatosis. The level of ADAM9 expression exerts influence on the tumor microenvironment. 

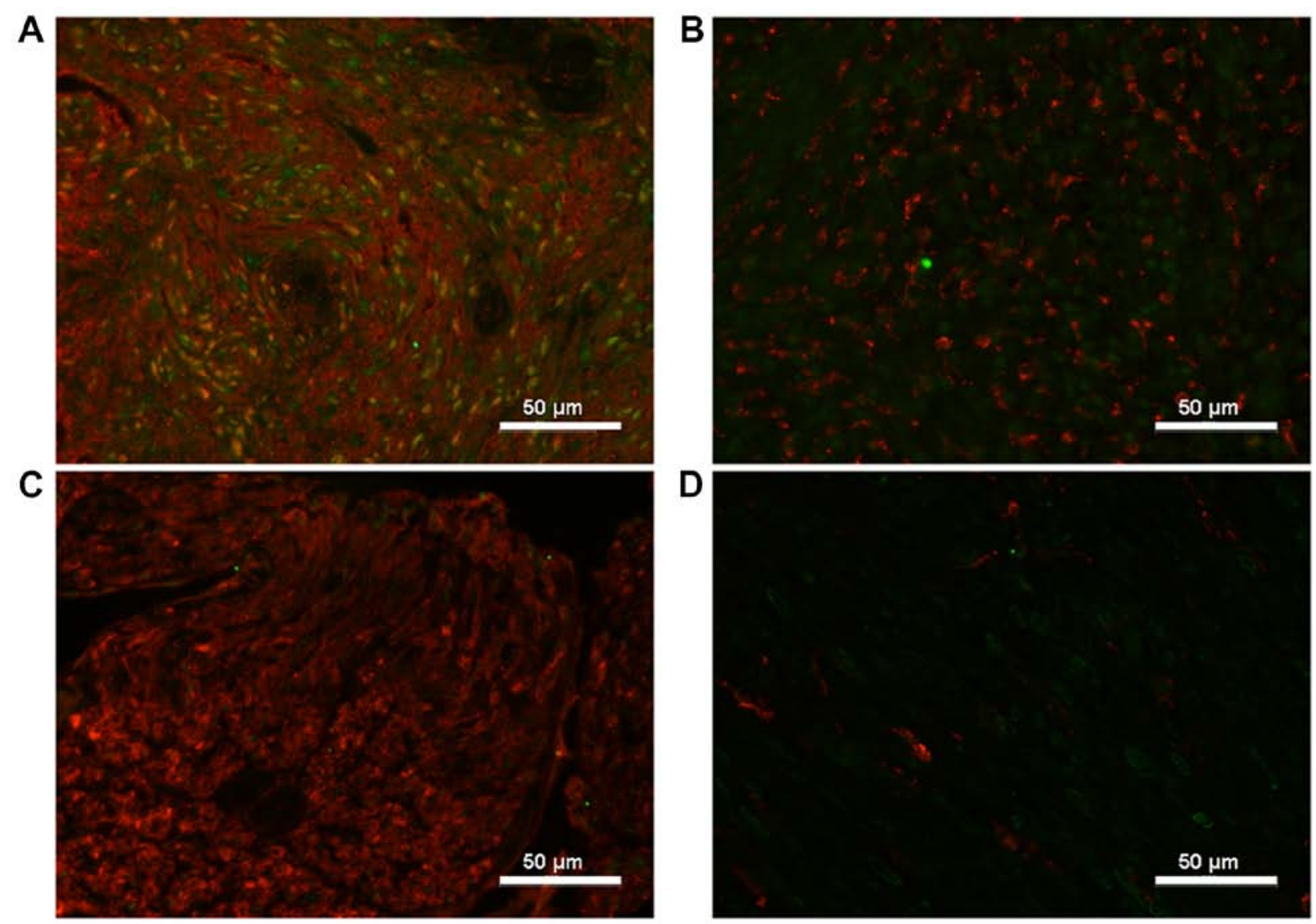

Figure 5. ADAM9 expression in paraffin-embedded sections of a representative vestibular schwannoma sample. (A) ADAM9 (green) co-localized with the Schwann cell/tumor marker S100 (red). (B) ADAM9 (green) did not co-localize with the macrophage marker CD68 (red). (C) Healthy vestibular nerves obtained from autopsies were positive for S100 (red), but did not express ADAM9 (green) and (D) only rarely CD68 (red). Scale bar, $50 \mu \mathrm{m}$. ADAM9, a disintegrin and metalloproteinase 9.

The disintegrin component of ADAM9 effectively attracts neutrophils, which promote tumor growth by CXCR2 activation and the neutrophil elastase release for example in lung cancer (32-34). Because of this effect and the other described protein functions of ADAM9 like the cell-cell and cell-matrix adhesion, it could be a promising target for a new systemic therapeutic intervention. ADAM proteins can be 'targeted' by MMP inhibitors, specific inhibitors, antibodies, TIMPs and prodomains of the protein $(10,14,20)$. In case of ADAM9 only antibody treatment and the use of prodomains is feasible (14) because no elective inhibitor exists so far and TIMPs do not work $(10,14)$. Specific inhibitors of other ADAM proteins (INCB3619) in combination with different chemotherapeutics like gefitinib or paclitaxel were effective in the treatment of several tumor types like non-small cell lung cancer or breast cancer, without severe side effects like musculoskeletal problems seen withMMP inhibitors (10). Therefore, ADAM9 might be a very promising new therapeutic target for the systemic treatment of VS $(10,11,14)$.

ADAM9 was overexpressed specifically by Schwann cells of VS, but not of normal nerves. Its expression was higher in sporadic cases compared to NF2-associated VS. ADAM9 expression levels, especially of the secreted isoform, significantly correlated with hearing loss of the patients and therefore could be a marker for invasiveness of the tumor growth.

\section{Acknowledgements}

The authors would like to thank Mrs. Siglinde Kühnel (technical assistant, University Hospital in Würzburg) and Mrs. Elisabeth Karl (technical assistant, University Hospital in Würzburg) for their technical assistance. The authors would also like to thank Dr Jose M. Perez (Department of Neurosurgery, University Hospital Würzburg) for providing samples and Professor Ralf-Ingo Ernestus (Department of Neurosurgery, University Hospital Würzburg) for supporting this work.

\section{Funding}

The present study was funded by the Interdisciplinary Center of Clinical Research (IZKF) Würzburg (Z-2/72) and the University of Würzburg in the funding program Open Access Publishing.

\section{Availability of data and materials}

The datasets used and/or analyzed during the present study are available from the corresponding author on reasonable request.

\section{Authors' contributions}

$\mathrm{MB}, \mathrm{AFK}, \mathrm{CM}, \mathrm{ML}$ and $\mathrm{CH}$ participated in the design of the study. MB, AS and DDM performed the experiments and, together with CMM, ML and $\mathrm{CH}$, performed the data analysis 
and interpretation. All authors contributed to data interpretation, drafting or revising the article, gave final approval of the version to be published, and agreed to be accountable for all aspects of the work.

\section{Ethics approval and consent to participate}

The study was approved by the local ethics committee of the University Hospital Würzburg with the statement number 145/16 in July 2016 and written informed consent was obtained from the patients for the use of their tissue and participation.

\section{Patient consent for publication}

All patients provided consent for publication.

\section{Competing interests}

The authors declare that they have no competing interests.

\section{References}

1. Stemmer-Rachamimov AO, Louis DN, Gunnlaugur PN, Antonescu CR, Borowsky AD, Bronson RT, Burns DK, Cervera P, McLaughlin ME, Reifenberger G, et al: Comparative pathology of nerve sheath tumors in mouse models and humans. Cancer Res 64: 3718-3724, 2004.

2. Louis DN, Ohgaki H, Wiestler OD and Cavenee WK: WHO Classification of tumours of the central nervous system. WHO/IARC Classification of tumours, 4th edition revised, Vol 1, 2016.

3. Lim SH, Ardern-Holmes S, McCowage G and de Souza P: Systemic therapy in neurofibromatosis type 2. Cancer Treat Rev 40: 857-861, 2014.

4. Hanemann CO: Magic but treatable? Tumours due to loss of merlin. Bain 131: 606-615, 2008.

5. Hexter A, Jones A, Joe H, Heap L, Smith MJ, Wallace AJ, Halliday D, Parry A, Taylor A, Raymond L, et al: Clinical and molecular predictors of mortality in neurofibromatosis 2: A UK national analysis of 1192 patients. J Med Genet 52: 699-705, 2015

6. Cooper J and Giancotti FG: Molecular insights into NF2/Merlin tumor suppressor function. FEBS Lett 588: 2743-2752, 2014.

7. Asthagiri AR, Parry DM, Butman JA, Kim HJ, Tsilou ET, Zhuang Z and Lonser RR: Neurofibromatosis type 2. Lancet 373: 1974-1986, 2009.

8. Schulz A, Zoch A and Morrison H: A neuronal function of the tumor suppressor protein merlin. Acta Neuropathol Commun 2: 82, 2014.

9. Schulz A, Büttner R, Hagel C, Baader SL, Kluwe L, Salamon J, Mautner VF, Mindos T, Parkinson DB, Gehlhausen JR, et al: The importance of nerve microenvironment for schwannoma development. Acta Neuropathol 132: 289-307, 2016.

10. Duffy MJ, McKiernan E, O’Donovan N and McGowan PM: Role of ADAMs in cancer formation and progression. Clin cancer Res 15: 1140-1144, 2009.

11. Klein T and Bischoff R: Active metalloproteases of the A disintegrin and metalloprotease (ADAM) family: Biological function and structure. J Proteome Res 10: 17-33, 2011.

12. Weskamp G, Kratzschmar J, Reid MS and Blobel CP: MDC9, a widely expressed cellular disintegrin containing cytoplasmic SH3 ligand domains. J Cell Biol 132: 717-726, 1996.

13. McKie N, Dallas DJ, Edwards T, Apperley JF, Russell RG and Croucher PI: Cloning of a novel membrane-linked metalloproteinase from human myeloma cells. Biochem J 318: 459-462, 1996.

14. Mochizuki S, Okada Y: ADAMs in cancer cell proliferation and progression. Cancer Sci 98: 621-628, 2007.

15. Fan X, Wang Y, Zhang C, Liu L, Yang S, Wang Y, Xing L, Qian Z, Fang S, Qiao H and Jiang T: ADAM9 expression is associated with glioma tumor grade and histological type, and acts as a prognostic factor in lower-grade gliomas. Int J Mol Sci 17: pii: E1276, 2016.

16. Huang CF, Yang SF, Chiou HL, Hsu WH, Hsu JC, Liu CJ and Hsieh YH: Licochalcone A inhibits the invasive potential of human glioma cells by targeting the MEK/ERK and ADAM9 signaling pathways. Food Funct 9: 6196-6204, 2018.
17. Oria VO, Lopatta P, Schmitz T, Preca BT, Nyström A, Conrad C, Bartsch JW, Kulemann B, Hoeppner J, Maurer J, et al: ADAM9 contributes to vascular invasion in pancreatic ductal adenocarcinoma. Mol Oncol 13: 456-479, 2019.

18. Mazzocca A, Coppari R, De Franco R, Cho JY, Libermann TA, Pinzani $\mathrm{M}$ and Toker A: A secreted form of ADAM9 promotes carcinoma invasion through tumor-stromal interactions. Cancer Res 65: 4728-4738, 2005.

19. Fry JL and Toker A: Secreted and membrane-bound isoforms of protease ADAM9 have opposing effects on breast cancer cell migration. Cancer Res 70: 8187-8198, 2010.

20. Qian LW, Mizumoto K, Urashima T, Nagai E, Maehara N, Sato N, Nakajima M and Tamaka M: Radiation-induced increase in invasive potential of human pancreatic cancer cells and its blockade by a matrix metalloproteinase inhibitor, CGS27023. Clin Cancer Res 8: 1223-1227, 2002.

21. Mongaret C, Alexandre J, Thomas-Schoemann A, Bermudez E, Chéreau C, Nicco C, Goldwasser F, Weill B, Batteux F and Lemare F: Tumor invasion induced by oxidative stress is dependent on membrane ADAM 9 protein and its secreted form. Int J Cancer 129: 791-798, 2011.

22. Shigemura K, Sung SY, Kubo H, Arnold RS, Fujisawa M, Gotoh A, Zhau HE and Chung LW: Reactive oxygen species mediate androgen receptor- and serum starvation-elicited downstream signaling of ADAM9 expression in human prostate cancer cells. Prostate 67: 722-731, 2007.

23. Breun M, Schwerdtfeger A, Martellotta DD, Kessler AF Perez JM, Monoranu CM, Ernestus RI, Matthies C, Löhr M and Hagemann C: CXCR4: A new player in vestibular schwannoma pathogenesis. Oncotarget 9: 9940-9950, 2018.

24. Hsia HE, Tüshaus J, Brummer T, Zheng Y, Scilabra SD and Lichtenthaler SF: Functions of 'A disintegrin and metalloproteases (ADAMs)' in the mammalian nervous system. Cell Mol Life Sci 76: 3055-3081, 2019.

25. Wippold FJ II, Lubner M, Perrin RJ, Lämmle M and Perry A: Neuropathology for the neuroradiologist: Antoni A and Antoni B tissue patterns. AJNR Am J Neuroradiol 28: 1633-1638, 2007.

26. Samii M and Matthies C: Management of 1000 vestibular schwannomas (acoustic neuromas): Hearing function in 1000 tumor resections. Neurosurgery 40: 248-262, 1997.

27. Hummel M, Perez J, Hagen R, Gelbrich G, Ernestus RI and Matthies C: When does hearing loss occur in vestibular schwannoma surgery? Importance of auditory brainstem response changes in early postoperative phase. World Neurosurg 95: 91-98, 2016.

28. Moffat DA, Kasbekar A, Axon PR and Lloyd SK: Growth characteristics of vestibular schwannoma. Otol Neurotol 33: 1053-1058, 2012.

29. Sughrue ME, Kane AJ, Kaur R, Barry JJ, Rutkowski MJ, Pitts LH, Cheung SW and Parsa AT: A prospective study of hearing preservation in untreated vestibular schwannomas. J Neurosurg 114: 381-385, 2011.

30. Livak KJ and Schmittgen TD: Analysis of relative gene expression data using real-time quantitative PCR and the 2(-Delta Delta C(T)) method. Methods 25: 402-408, 2001.

31. Oria VO, Lopatta $\mathrm{P}$ and Schilling $\mathrm{O}$ : The pleiotropic role of ADAM9 in the biology of solid tumors. Cell Mol Life Sci 75: 2291-2301, 2018

32. Heit B, Tavener S, Raharjo E and Kubes P: An intracellular signaling hierarchy determines direction of migration in opposing chemotactic gradients. J Cell Biol 159: 91-102, 2002.

33. Amendola RS, Martin AC, Selistre-de-Araújo HS, Paula-Neto HA, Saldanha-Gama R and Barja-Fidalgo C: ADAM9 disintegrin domain activates human neutrophils through an autocrine circuit involving integrins and CXCR2. J Leukoc Biol 97: 951-962, 2015.

34. Gong L, Cumpian AM, Caetano MS, Ochoa CE, De la Garza MM, Lapid DJ, Mirabolfathinejad SG, Dickey BF, Zhou Q and Moghaddam SJ: Promoting effect of neutrophils on lung tumorigenesis is mediated by CXCR2 and neutrophil elastase. Mol Cancer 12: 154, 2013.

This work is licensed under a Creative Commons Attribution-NonCommercial-NoDerivatives 4.0 International (CC BY-NC-ND 4.0) License. 